Cuadernos de Filología Clásica. Estudios Latinos

ISSN: 1131-9062

https://dx.doi.org/10.5209/cfcl.64890

\title{
La difficile ars epigrammatica di Marziale
}

\author{
Maria Salanitro ${ }^{1}$
}

Recibido: 27/10/2018 / Aceptado: 24/11/2018

Resumen. Marziale è un epigrammista molto acuto e la sua raffinata arguzia, unita alla sinteticità dello stile, può trarre in inganno i moderni interpreti. Così le traduzioni che rivelano, senza possibilità di dubbio, le interpretazioni, si trasformano in un tradimento nei confronti del poeta. Questo accade nel gruppo di epigrammi del decimo libro in cui il poeta annuncia ai suoi lettori l'intenzione di ritornare a Bilbilis, la città spagnola in cui era nato. Particolarmente sorprendenti sono gli errori di interpretazione di 10.37 e 10.104 .

Parole chiave: Marziale; Roma; Callaïcum; ad Oceanum; Augusta Bilbilis; non moratur.

\section{[en] The difficult epigrammatic ars of Martial}

\begin{abstract}
Martial is a very acute epigrammist and his refined wit, combined with the conciseness of style, can mislead modern interpreters. Therefore the translations that reveal the interpretations become a sort of betrayal of the poet. This is what happens in the group of epigrams of the tenth book, in which the poet announces to his readers the intention to return to Bilbilis, the Spanish city where he was born. The interpretative errors of 10.37 and 10.104 are particularly surprising.
\end{abstract}

Keywords: Martial; Rome; Callaïcum; ad Oceanum; Augusta Bilbilis; non moratur.

Sumario. 1. Introduzione. 2. Mart.3.33.3. Mart.5.63. 4. Mart.5.66. 5. Mart.10.13(20). 6. Mart.10.37. 7. Mart.10.78. 8. Mart.10.92. 9. Mart.10.96. 10. Mart.10.103. 11. Mart.10.104

Cómo citar: Salanitro, M., «La difficile ars epigrammatica di Marziale», en Cuad. Filol. Clás. Estud. Lat. 39.1 (2019), 29-41.

\section{Introduzione}

«L'epigramma di Marziale, nonostante la molteplicità dei motivi lirici, resta modello insuperato del componimento poetico breve, acuto, veloce, che abbia alla fine aliquid luminis». Così scriveva Concetto Marchesi $(1952,140)$ che traduceva l'espressione aliquid luminis usata da Frontone per definire la legge dell'epigramma, con 'il razzo finale'. Ma va evidenziato che la chiusa degli epigrammi di Marziale è, spesso, così raffinata e preziosa da mettere a dura prova l'acume dei lettori.

Ne presento qui alcuni esempi che si aggiungono ai molti altri di cui mi sono occupata durante il mio studio costante dell'opera di Marziale.

«Sapienza» Università di Roma.

E-mail: mariasalanitro@virgilio.it 


\section{MART.3.33}

Ingenuam malo, sed si tamen illa negetur,

libertina mihi proxuma condicio est:

extremo est ancilla loco: sed uincet utramque,

si facie nobis haec erit ingenua.

Nell'interpretazione della pointe gli studiosi del nostro poeta si sono trovati in difficoltà. Nella prima metà del Novecento Izaac $(1930,93)$ tradusse: «mais elle l'emportera sur l'une et sur l'autre si elle a un joli minois». In tempi più vicini ai nostri due studiosi italiani hanno tradotto: «ma se il suo viso sarà per me quello di una donna di liberi natali, supererà l'una e l'altra» (Norcio 1980, 249); «ma le vincerà entrambe se sarà graziosa di viso» (Fusi 2006, 281).

E facile capire che $\mathrm{i}$ traduttori moderni, trovandosi in difficoltà, hanno ricalcato l'interpretazione di Izaac, che è discutibile non solo per il senso, ma anche perché lo studioso è stato costretto, nella speranza di fornire un significato accettabile, a non prendere in considerazione il pronome nobis. Tutto ciò ci dice che va cercata un'altra spiegazione.

$\mathrm{Ci}$ aiuta a capire la consapevolezza che l'aggettivo (ingenua) che apre l'epigramma non può avere il significato che ha quello in chiusura. È un procedimento che troviamo, ad es., in un epigramma del primo libro:

«Liber homo es nimium», dicis mihi, Ceryle, semper.

In te qui dicit, Ceryle, liber homo est. (MART.1.67)

L'epigramma è fondato sul doppio senso di liber. Nella narratio apprendiamo che Cerilo, personaggio di origine servile, come rivela il suo nome, rivolge al poeta l'accusa di essere nimium liber, cioè «troppo sfacciato». Nel secondo verso liber è usato nel suo significato primario. In te, posto all'inizio del verso, prepara con tutta la sua carica aggressiva la manifestazione dell'esplicito disprezzo del povero, ma liber poeta nei confronti del ricco, ma servus Cerilo: «Colui che parla contro di te, o Cerilo, è un uomo libero».

Nel nostro epigramma ingenua del primo verso significa «libera», mentre nella seconda occorrenza il poeta utilizza un significato secondario, «raffinata», e lo stesso accade per il sostantivo facies che qui non vuol dire 'viso', ma 'aspetto'. Dobbiamo quindi tradurre: «ma supererà entrambe, se avrà per me un aspetto raffinato». La validità di questa interpretazione mi sembra confermata dal fatto che ci permette di tradurre il termine che nell'interpretazione 'tradizionale' doveva essere necessariamente sottaciuto, cioè nobis che è un datiuus iudicantis.

Questo epigramma, come il precedente, è un caso esemplare della difficoltà del tradurre: noi siamo costretti a tradurre ingenua del primo verso con un significante («libera») che non coincide con quello con cui dobbiamo tradurre il secondo ingenua («raffinata»). Si perde così il gioco di conformità del significante nella disformità del significato che si realizza nel testo latino. 


\section{MART.5.63}

«Quid sentis» inquis «de nostris, Marce, libellis?»

Sic me sollicitus, Pontice, saepe rogas.

Admiror, stupeo: nihil est perfectius illis,

ipse tuo cedet Regulus ingenio.

«Hoc sentis?» inquis «faciat tibi sic bene Caesar,

sic Capitolinus Iuppiter». Immo tibi.

Un benemerito studioso dell'opera di Marziale, Alberto Canobbio (2011, 492), così spiega il significato dell'epigramma: «Pontico insiste per sapere quale sia la vera opinione che, a proposito dei suoi componimenti, ha M. (vv. 1-2), ma quest'ultimo, dopo aver espresso la sua in realtà assente ammirazione per il poetastro mediante delle improbabili iperboli (vv. 3-4), è così determinato a lusingare il suo interlocutore che rimanda al mittente anche un buon augurio».

Questa interpretazione coincide con quella dei predecessori da lui citati (Friedlaender, Izaac, Shackleton Bailey e Howell).

È uno dei casi, non infrequenti, in cui ogni interprete, trovandosi in difficoltà, si è limitato ad 'appoggiarsi' agli studiosi che lo hanno preceduto. Così uno dei più notevoli esempi del ricorso del nostro poeta all'ả finale anodino. Per capire il significato dobbiamo esaminare la costruzione dell'epigramma.

Dai primi due versi apprendiamo che Pontico chiede spesso ansiosamente a Marziale che cosa pensi delle sue poesie. La risposta, contenuta nei due versi seguenti, è tale da far credere che il giudizio di Marziale sia altamente positivo anche se l'esagerazione degli elogi in crescendo (Admiror, stupeo: nihil est perfectius illis, / ipse tuo cedet Regulus ingenio) fa nascere qualche dubbio sulla sincerità del poeta. Nel quinto verso e in gran parte del sesto le parole augurali di Pontico, scaturite da uno slancio di gratitudine («così sia benevolo con te l'imperatore, così Giove Capitolino») ribaltano la situazione e confermano il lettore nella prima impressione che il giudizio sia positivo. Ma ecco, inattese, le due sferzanti parolette finali: Immo tibi («anzi a te») che vengono a significare «sei tu, povero imbrattacarte, che hai bisogno della benevolenza del dio terreno e di quello celeste».

\section{MART.5.66}

Saepe salutatus numquam prior ipse salutas:

sic eris Aeternum, Pontiliane, Vale.

Così si legge nell' edizione di Izaac (1930, 170). Prima di lui Friedlaender (1886, 422) aveva così interpunto il secondo verso: sic eris "Aeternum», Pontiliane, «uale $»^{2}$. La sua scelta è stata condivisa dagli altri editori con l'eccezione di Alberto Canobbio $(2011,512)$, che, inopinabilmente, ha scelto di porre un pun-

2 Lo studioso tedesco segnala di aver adottato l'interpunzione di Gilbert. 
to interrogativo dopo eris, accogliendo una proposta di Housman ${ }^{3}$ che non è stata presa in considerazione da nessun editore perché oscura il significato del monodistico, come risulta dalla traduzione di Canobbio: "Anche se ti ho salutato spesso tu non saluti mai per primo: continuerai a comportarti così? Addio per sempre, Pontiliano». Questa interpretazione ha i piedi di argilla. Anzitutto, non si può piegare il verbo sum al significato, non documentato, di «continuo a comportarmi»e, in secondo luogo, segnando un punto interrogativo dopo eris, si determina un contrasto fra l'interrogativa che presuppone l'attesa di un comportamento diverso, e la decisa repulsa del finale.

In realtà, il primo verso contiene la narratio, il secondo la pointe, come segnalano i due punti a chiusura del primo verso segnati da tutti gli editori compreso lo stesso Canobbio.

Va precisato che Friedlaender scelse di chiudere tra virgolette sia Aeternum che Vale perché si accorse, come evidenziò in una nota, che c'è un rimando a un' espressione usata da Virgilio in Aen. 11.98. È facile constatare che la pointe sarebbe banale se non fosse 'nobilitata' dall'allusione a un verso virgiliano.

Sono numerosi gli epigrammi in cui Marziale riutilizza la poesia virgiliana ${ }^{4} \mathrm{o}$ con intenti celebrativi o per equiparare qualche suo personaggio a uno descritto da Virgilio ${ }^{5}$ o per ribaltare scherzosamente qualche epiteto ${ }^{6}$ o anche con intenti parodici. Nel nostro caso l'allusione è dissacrante perché evoca un contesto pervaso da intensa commozione. Enea, gemendo, rivolge l'estremo saluto a Pallante (Aen.11.97-98): salue aeternum mihi, maxime Palla, / Aeternumque uale. Virgilio costruisce il pathos con l'attribuzione dell'aggettivo maxime a Pallante e con l'iterazione delle formule di addio in chiasmo (salue aeternum ... Aeternumque uale).

La sincera ammirazione di Marziale nei confronti di Virgilio ${ }^{7}$ non esclude puntate irriverenti dettate da un atteggiamento polemico nei confronti dei generi elevati.

Per quanto riguarda il testo del nostro epigramma si può accettare quello di Friedlaender, ma non è necessario chiudere tra virgolette aeternum e uale, altrimenti dovremmo riservare lo stesso trattamento a tutti i luoghi che alludono alle opere virgiliane. Infine aeternum e uale vanno scritti con l'iniziale minuscola come nel verso di Virgilio:

Saepe salutatus numquam prior ipse salutas:

sic eris aeternum, Pontiliane, uale.

«Spesso salutato non mi saluti mai per primo:

così sarai per me un addio eterno».

3 Housman fu un congetturatore geniale, ma talora troppo audace anche per il suo disprezzo della mediocrità del 'metodo' non accompagnato dall'ingegno, $c f$. Timpanaro $(1978,147)$.

$4 \quad C f$. Citroni (1987, 399); Salanitro (2005, 1-14, ora anche in Salanitro 2012, 221-235).

5 Ad es. in 6.64.27 l'espressione rabido ore, riferita nell'Eneide ad Alletto, serve ad evidenziare la rabbiosa aggressione di un detrattore.

6 In 12.63.3 il fiume Galeso è definito albus in giocoso contrasto con il niger usato da Virgilio in Georg.4.126, cf. La Penna (1983, 160).

$7 \quad$ Il nostro poeta lo definisce magnus $(4.14 .14 ; 11.48 .1 ; 12.67 .5)$ e summus $(12.3[4] .1)$. 
Izaac $(1930,267)$ chiosava: «C'est la formule du salut aux morts et cela veut dire: tu n'existeras plus à mes yeux». È così. L'ultimo verso viene a significare: «Tu sarai per me un morto» ${ }^{8}$.

\section{MART.10.13(20)}

La morte di Domiziano, avvenuta nel settembre del 96, cambiò la vita di Marziale. Il tentativo di ingraziarsi i successori, Nerva e Traiano, fallì perché il poeta si era compromesso sia celebrando in molti epigrammi l'imperatore Domiziano sia frequentando i suoi cortigiani.

Il poeta si rese conto che a Roma non c'era più spazio per lui e si preparò al suo ritorno in patria. Possiamo seguire le fasi del distacco da Roma in alcuni epigrammi della seconda edizione del decimo libro approntata nel $98^{9}$. Il primo epigramma in cui il poeta annuncia ai suoi lettori l'intenzione di ritornare in Spagna è il 13 (20):

Ducit ad auriferas quod me Salo Celtiber oras, pendula quod patriae uisere tecta libet,

tu mihi simplicibus, Mani, dilectus ab annis

et praetextata cultus amicitia,

tu facis; in terris quo non est alter Hiberis dulcior et uero dignus amore magis.

Tecum ego uel sicci Gaetula mapalia Poeni et poteram Scythicas hospes amare casas.

Si tibi mens eadem, si nostri mutua curast, in quocumque loco Roma duobus erit.

L'epigramma non presenta problemi esegetici e non ha particolari pregi.

Nei due versi iniziali viene presentata la piccola città del poeta, Bilbilis, con due peculiari caratteristiche topografiche, come accade in altri epigrammi: il fiume Salone che scorre ai suoi piedi e l'arroccamento sul costone di un monte, qui espresso con un'efficace perifrasi poetica, pendula tecta ${ }^{10}$. Nei vv. 3-4 la tensione si sposta e si concentra sul protagonista, Manio, concittadino e amico fin dagli anni dell'infanzia. Nei versi seguenti le manifestazioni di fiducia e di affetto si susseguono in climax. Il nono verso prepara la pointe che abbraccia, cosa che accade raramente, gli ultimi due versi: «se tu hai la stessa inclinazione e se ricambi il mio affetto, Roma sarà per noi due in qualunque luogo».

8 Nel nostro Meridione ci si separa da una persona che ci ha fatto dei torti con la frase «per me sei morto» o semplicemente «sei morto». Molto divertente la disavventura di un politico campano che, avendo rotto i rapporti con un amico non conterraneo, andava dicendo di lui: «è morto». L'espressione fu fraintesa e il politico dovette difendersi dall'accusa di minaccia di morte.

9 Non possediamo la prima edizione che risaliva al 95. Della revisione ci dà notizia lo stesso Marziale nei primi quattro versi del secondo epigramma del libro: Festinata prior, decimi mihi cura libelli / elapsum manibus nunc reuocauit opus. / Nota leges quaedam, sed lima rasa recenti; / pars noua maior erit. Apprendiamo così che alcuni epigrammi sono stati ritoccati, ma la maggior parte sono nuovi. La motivazione del rifacimento (Festinata prior... cura) non è veritiera: è facile intuire che si rese necessario per l'eliminazione di alcuni epigrammi compromettenti, fra cui quelli che riguardavano Domiziano.

10 In 1.49.3 e in 10.104.4 darà a Bilbilis l'epiteto di alta che ha anche una valenza nobilitante. 
I commentatori evidenziano che questo è il primo epigramma in cui Marziale annuncia ai suoi lettori il proposito di ritornare in Spagna. Ma va anche notato che qui il poeta riconosce quanto importante sia stato per lui il lungo soggiorno a Roma, la città che ha ispirato $\mathrm{i}$ suoi versi e gli ha dato la fama ${ }^{11}$.

\section{MART.10.37}

Iuris et aequarum cultor sanctissime legum, ueridico Latium qui regis ore forum, municipi, Materne, tuo ueterique sodali Callaïcum mandas si quid ad Oceanum...-

An Laurentino turpis in litore ranas et satius tenues ducere credis acos ad sua captiuum quam saxa remittere mullum, uisus erit libris qui minor esse tribus.

Et fatuam summa cenare pelorida mensa quosque tegit leui cortice concha breuis

ostrea Baianis quam non liuentia testis, quae domino pueri non prohibente uorent?

Hic olidam clamosus ages in retia uulpem mordebitque tuos sordida praeda canes:

Illic piscoso modo uix educta profundo inpedient lepores umida lina meos. -

Dum loquor ecce redit sporta piscator inani, uenator capta maele superbus adest:

omnis ab urbano uenit ad mare cena macello. Callaïcum mandas si quid ad Oceanum...

Devo subito precisare che ho eliminato il punto interrogativo che chiude il v. 8 in tutte le edizioni. Il primo ad accorgersi che il punto interrogativo non era accettabile è stato Claudio Buongiovanni $(2012,209)$ che ha spiegato così la sua scelta: «Al v. 8 si è preferito porre una virgola in luogo del punto interrogativo; tale scelta sembra rendere al meglio non solo il lungo 'catalogo oppositivo' presentato da M., che risulterebbe ingiustamente spezzato e indebolito da un'interpretazione troppo forte». Certamente il punto interrogativo va eliminato, ma senza alcuna sostituzione, perché anche la virgola prima della congiunzione coordinante non si giustifica. Accade, come ho messo in rilievo in varie occasioni, che i traduttori scelgano la giusta interpunzione, in contrasto con il testo adottato. Così Giuseppe Norcio, pur in presenza di un punto interrogativo nel testo critico da lui adottato, quello di Giarratano, ha sentito, quasi istintivamente, che non si poteva interrompere il necessario continuum e ha eliminato ogni segno di interpunzione dopo la parola finale del v. 8 (tribus).

11 Nel primo periodo del suo soggiorno a Roma il poeta vedeva la città come un luogo in cui era difficile vivere perché non si trovava alcun lavoro remunerativo e anche l'umile mestiere di cliente assicurava guadagni a pochi. Così, a conclusione dell'epigramma 3.38, diceva a un finto interlocutore: si bonus es, casu uiuere, Sexte, potes. Ma, non molti anni dopo cambiò opinione, al punto da sentire Roma come la sua città: Laudat, amat, cantat nostros mea Roma libellos (6.60[61].1). Sull'amore-odio per Roma vd. Parroni (1984). 
Sull'ultimo verso di questo epigramma, cioè sulla pointe, nulla dicono i commentatori. Buongiovanni $(2012,188)$ ritiene che il «Witz explicitario»s si trova nel penultimo verso come «degna conclusione del messaggio costruito nell'intero componimento che assolve funzioni di graffiante e disillusa $\sigma \varphi \rho \alpha \gamma i$ comica». Si resta molto perplessi perché è noto che il Witz in tutti i componimenti epigrammatici si esplicita o nell'intero verso finale o nel secondo emistichio dello stesso o nell'ultima parola ${ }^{12}$. E, d'altra parte, se, per assurdo, collocassimo l'arguzia nel penultimo verso, che senso avrebbe il nostro verso finale? Buongiovanni $(2012,232)$ spiega: «Ora M., dopo le immagini simboliche del pescator [sic!] e del venator, si rende conto che Materno è ormai rassegnato e quindi perduto, e allora riprende il discorso dove lo ha lasciato, ma non lo completa perché sa che è inutile».

Per capire dobbiamo esaminare la struttura dell'epigramma. Dai primi due versi apprendiamo che il poeta si rivolge a un personaggio importante, che «guida il foro latino». Il terzo verso ci rivela che il personaggio si chiama Materno ed è un concittadino e un amico di vecchia data del poeta ${ }^{13}$.

Il quarto verso (Callaïcum mandas si quid ad Oceanum) viene unanimemente tradotto «Se hai qualche commissione da dare al tuo compatriota e amico per l'Oceano della Galizia». Ma che significa "per l'Oceano della Galizia»? Questa traduzione non è autorizzata né dalla sintassi (ad Oceanum è complemento di moto a luogo) né dal buon senso perché non si danno commissioni a un mare.

Chi ha studiato l'opera di Marziale sa che il poeta ricorre spesso a espressioni ellittiche. Qui bastino due significativi esempi. In II 29 il poeta si sofferma sulla straordinaria cura, nella persona e nell'abbigliamento, di Rufo. Il v. 5 (cuius olet toto pinguis coma Marcelliano) crea grande difficoltà agli interpreti per l'estrema concisione. Noi dobbiamo esplicitare tutto ciò che il poeta ha taciuto traducendo: «La cui chioma è impomatata con tutti i tipi di profumo che emanano dagli spettatori del teatro di Marcello» e ancora l'espressione lateris frigora trita tui (II 46, 8) richiede una traduzione che spieghi tutto ciò che è implicito: «La fredda logora toga di chi cammina al tuo fianco» ${ }^{14}$.

Più semplice il verso del nostro epigramma, in cui è taciuto soltanto il participio eunti da concordare con municipi... tuo ueterique sodali: «Se hai qualche commissione da dare al tuo concittadino e amico che va nei pressi dell'Oceano della Galizia.... $\rangle^{15}$, cioè nella Spagna Tarraconese dove si trova Bilbilis.

Nei vv. 5-16 il poeta crea con vari esempi una contrapposizione delle scarse risorse marine e terrestri della villa Laurentina di Materno ai cibi abbondanti e pregiati offerti dal mare e dalla terra di Spagna.

Il distico seguente (vv. 17-18) è una conferma della veridicità delle asserzioni precedenti: il poeta si trova nella villa di Materno e può constatare che il pescatore torna con il paniere vuoto e il cacciatore torna tutto tronfio (superbus) per il fatto che

12 Rari i casi in cui l'arguzia abbraccia i due versi finali.

13 A Materno Marziale si rivolge anche in 1.96 e in 2.74. Era un avvocato illustre, con ogni probabilità insignito del ius publicae respondendi, cf. Citroni $(1975,294)$.

14 La sinteticità dello stile del nostro poeta rende difficile, come abbiamo visto, anche la comprensione della pointe di alcuni epigrammi.

15 Ritengo che dopo Galizia e prima della barretta, che segnala l'inizio dell'ampio inciso, debbano essere segnati dei puntini di sospensione che sottendono l'apodosi del periodo ipotetico: «Io sono a tua disposizione». Così anche nel verso finale. 
può esibire un tasso, cibo poco appetibile ${ }^{16}$. Si raggiunge così la climax nella descrizione della disastrosa mancanza di cibi nella villa, e il verso seguente, il penultimo, è la naturale conseguenza della climax: Materno per assicurarsi la cena deve ricorrere al mercato della città ${ }^{17}$. Il verso finale è, a ragion veduta, identico al v. 4 . Il poeta ribadisce la sua offerta ma, dopo la lunga descrizione delle misere risorse della villa, l'offerta diventa ironica e provocatoria, perché Materno non ha bisogno di qualcosa (quid), Materno ha bisogno di tutto. Naturalmente, chi traduce l'epigramma non deve dimenticare che l'ultimo verso va inteso così: «Se hai qualche commissione da dare a uno che va nei pressi dell'Oceano della Galizia».

\section{MART.10.78}

Ibis litoreas, Macer, Salonas, ibit rara fides amorque recti et quae, cum comitem trahit pudorem, semper pauperior redit potestas: felix auriferae colone terrae, rectorem uacuo sinu remittes optabisque moras, et exeuntem udo Dalmata gaudio sequeris. Nos Celtas, Macer, et truces Hiberos cum desiderio tui petemus.

Sed quaecumque tamen feretur illinc piscosi calamo Tagi notata, Macrum pagina nostra nominabit: sic inter ueteres legar poetas, nec multos mihi praeferas priores, uno sed tibi sim minor Catullo.

Il poeta sceglie come protagonista del suo epigramma l'amico Macro per un evento parallelo: mentre Macro, che è un dalmata, si accinge a partire per la sua terra con la carica di governatore, Marziale si accinge a tornare nella sua Spagna. Il poeta loda la lealtà, l'amore per la giustizia e l'onestà di Macro. Fortunata la Dalmazia che non sarà depredata, secondo l'uso corrente, dal suo governatore! Marziale promette a Macro, naturalmente esagerando, di nominarlo in tutti gli scritti che invierà dalla Spagna. L'epigramma si conclude con parole di speranza in quanto il poeta, come in parecchi epigrammi precedenti, si dichiara convinto della validità della sua poesia. I vv. 14-15 preparano la pointe: il poeta si augura che l'amico legga la sua opera come legge quelle degli antichi poeti e lo anteponga solo a pochi predecessori. Ma ecco, improvvisamente, nell'ultimo verso, un'affermazione che modifica il pensiero espresso nei due versi precedenti: Macro lo consideri inferiore al solo Catullo ${ }^{18}$.

16 Sembra che il tasso non fosse tra gli animali di cui abitualmente si nutrivano i Romani: si veda Braconi 2015.

17 Il verso riecheggia il penultimo verso di 3.58. Basso è costretto a portare nella sua villa dalla città holus, oua, pullos, poma, caseum, mustum.

18 L'accostamento dei suoi epigrammi a quelli di Catullo si trova già nell'ultimo epigramma del settimo libro (99) e sarà confermato nel penultimo epigramma del nostro libro (103). 


\section{MART.10.92}

Marri, quietae cultor et comes uitae, quo ciue prisca gloriatur Atina, has tibi gemellas barbari decus luci commendo pinus ilicesque Faunorum et semidocta uilici manu structas

Tonantis aras horridique Siluani, quas pinxit agni saepe sanguis aut haedi, dominamque sancti uirginem deam templi, et quem sororis hospitem uides castae Martem mearum principem Kalendarum, et delicatae laureum nemus Florae, in quod Priapo persequente confugit. Hoc omne agelli mite paruuli numen seu tu cruore siue ture placabis:

«Ubicumque uester Martialis est», dices, «Hac ecce mecum dextera litat uobis Apsens sacerdos; uos putate praesentem et date duobus quidquid alter optabit».

Un altro congedo dall'Italia, qui rappresentata dal piccolo podere di Nomento. La partenza è vicina e il poeta affida a Marrio, un carissimo amico, come risulta chiaramente dal primo verso, quello che possiamo definire l'angolo del sacrario del suo podere. Ci sono le are di Giove e di Silvano, un tempietto dedicato a Diana che ospita una statua di Marte e, infine, una statua di Flora inseguita da Priapo. E tutto ciò è inserito in un riposante ambiente agreste: due pini gemelli, cioè piantati contemporaneamente $^{19}$, all'interno di un bosco sacro, allo stato selvaggio ${ }^{20}$, alcuni lecci e un bosco di alloro.

In chiusura dell'epigramma la preghiera suggerita all'amico raggiunge la sua climax nel verso finale: «E concedete ad entrambi ciò che ognuno di noi chiederà». La consapevolezza che l'amico lo terrà presente nelle preghiere ai 'suoi' dèi, stempera il rimpianto per quel luogo di vita serena che non sarà più suo.

\section{Mart.10.96}

Saepe loquar nimium gentes quod, Auite, remotas

miraris, Latia factus in urbe senex,

auriferumque Tagum sitiam patriumque Salonem

et repetam saturae sordida rura casae.

Illa placet tellus in qua res parua beatum

19 I commentatori restano perplessi di fronte all'aggettivo gemellas che, riferito alle piante, è un hapax. Si suole tradurlo con «simili» (cf. ThlL s.u. «gemellus»). Io ritengo che Marziale, creatore di nuove immagini, abbia usato il termine per contiguità con la specie umana: i due pini sono nati (cioè piantati) contemporaneamente, come accade per i figli degli uomini.

20 Il poeta usa l'espressione barbari luci, che Scàndola $(1996,873)$ traduce giustamente «di una sacra boscaglia», poiché si tratta di un bosco non modificato dalla mano dell'uomo. 
me facit et tenues luxuriantur opes:

pascitur hic, ibi pascit ager; tepet igne maligno

hic focus, ingenti lumine lucet ibi;

hic pretiosa fames conturbatorque macellus,

mensa ibi diuitiis ruris operta sui;

Quattuor hic aestate togae pluresue teruntur, autumnis ibi me quattuor una tegit.

I, cole nunc reges, quidquid non praestat amicus

cum praestare tibi possit, Auite, locus.

È una uariatio di 10.37. Si ripete la contrapposizione tra la Spagna e Roma. La Spagna permette una vita semplice e assicura prodotti genuini e non costosi come quelli che si vendono a Roma. Questo è il contenuto di fondo, ma qui Marziale dormitat, le immagini sono scialbe e ripetitive. Emergono solo il quinto e il sesto verso: Illa placet tellus in qua res parua beatum / me facit et tenues luxuriantur opes («Mi piace quella terra in cui il poco mi rende felice e un modesto patrimonio è una ricchezza»). La pointe, banale, non riscatta l'intero componimento.

\section{MART.10.103}

Municipes, Augusta mihi quos Bilbilis acri monte creat, rapidis quem Salo cingit aquis, ecquid laeta iuuat uestri uos gloria uatis?

Nam decus et nomen famaque uestra sumus,

nec sua plus debet tenui Verona Catullo meque uelit dici non minus illa suum.

Quattuor accessit tricesima messibus aestas, ut sine me Cereri rustica liba datis, moenia dum colimus dominae pulcherrima Romae: mutauere meas Itala regna comas.

Excipitis placida reducem si mente, uenimus; aspera si geritis corda, redire licet.

Nell'imminenza della partenza il poeta appare timoroso perché non sa come lo accoglieranno i suoi concittadini.

Nell'epigramma è felicemente espresso l'alternarsi di pensieri contrastanti. Il poeta è in bilico tra la speranza e il dubbio.

Nel periodo che abbraccia i primi tre versi, dopo la parola iniziale che, non a caso, è municipes, e dopo la presentazione di Bilbilis, adagiata su un aspro monte intorno a cui scorre il vorticoso Salone, ecco una frase interrogativa da cui traspare l'incertezza del momento: il poeta si chiede se il suo ritorno sarà gradito ai suoi concittadini e spera che la sua fama li renda benevoli. Notevole, sul piano stilistico, il nesso allitterante uestri uos (v. 3), con cui si insiste sull'appartenenza del poeta alla sua città natale, un'appartenenza che nell'aspettativa del poeta doveva sfociare in una reciprocità. Quest'aspettativa ispira i tre versi seguenti (vv. 4-6) ed è evidenziata dal ricorso all'aggettivo uester che coinvolge tre termini (decus, nomen, fama) e accomuna il poeta ai suoi concittadini. Mar- 
ziale dice ai municipes: voi dovete accogliermi perché io sono il vostro vanto e la vostra fama, dovete rendermi omaggio come fa la città di Verona nei confronti di Catullo a cui io non sono inferiore.

L'autoelogio mira a rafforzare l'incerta speranza di una buona accoglienza. Segue la precisazione dei molti anni trascorsi lontano dalla patria, abbandonata per vivere nella città signora del mondo. Il dubbio sull'accoglienza ritorna nel penultimo verso e viene abilmente neutralizzato con il ricorso alla scherzosa minaccia dell'ultimo verso.

\section{MART.10.104}

I nostro comes, i, libelle, Flauo

longum per mare, sed fauentis undae,

et cursu facili tuisque uentis

Hispanae pete Tarraconis arces:

illinc te rota tollet et citatus

altam Bilbilin et tuum Salonem

quinto forsitan essedo uidebis.

Quid mandem tibi quaeris? Vt sodales

paucos, sed ueteres et ante brumas

triginta mihi quattuorque uisos

ipsa protinus a uia salutes

et nostrum admoneas subinde Flauum

iucundos mihi nec laboriosos

secessus pretio paret salubri,

qui pigrum faciant tuum parentem.

Haec sunt. Iam tumidus uocat magister

castigatque moras, et aura portum

laxauit melior: uale, libelle:

nauem, scis puto, non moratur unus.

È un'allocuzione a un suo libro che l'amico Flavo porta con sé a Bilbilis ${ }^{21}$.

Buongiovanni $(2012,428)$ ritiene che l'ultimo verso sia un detto squisitamente popolare non documentato in ambito letterario. Siamo di fronte a un'interpretazione molto fuorviante ${ }^{22}$. In realtà la pointe è del tutto perspicua tanto è vero che $\mathrm{i}$ commentatori non hanno sentito il bisogno di chiosarla.

La struttura dell'epigramma è molto semplice. Nel primo verso il poeta ordina al suo libro di partire in compagnia dell'amico Flavo. Il secondo e il terzo verso contengono un minuscolo $\pi \rho \circ \pi \varepsilon \mu \pi \tau \iota \kappa o ́ v$, l'augurio di un mare tranquillo ${ }^{23} \mathrm{e}$ di venti

21 Marziale si rivolge al suo libro in ben 17 epigrammi, $c f$. Citroni $(1986,136)$.

22 L'incomprensione genera altra incomprensione. Un'espressione trasparente come scis puto diventa un espediente stilistico che «ha il non semplice compito di smorzare i toni patetici dell'addio [...]; formalmente M. ottiene il suo obiettivo, ma paradossalmente genera nel lettore consapevole delle vicende successive una maggiore malinconica sympatheia» (Buongiovanni 2012, 424).

23 L'espressione longum per mare evidenzia tutta la distanza che separa Bilbilis da Roma. 
favorevoli. Poi, una volta approdato a Tarragona, il libro sarà trasportato da una carrozza a Bilbilis, connotata dalla sua posizione elevata e dal suo fiume, il Salone ${ }^{24}$.

Il poeta affida al libro il compito di salutare i pochi vecchi amici e di fare in modo che Flavo gli procuri un piacevole domicilio in cui il poeta, che attraverso l'espressione tuum parentem chiama il libro suo figlio, possa riposarsi (vv. 8-15). I versi seguenti preparano la pointe con l'informazione che il comandante della nave chiama e rimprovera il libro che si attarda. Infine Marziale saluta il libro e conclude: nauem, scis puto, non moratur unus, costruendo il verso più importante dell'epigramma con una considerazione dettata dal buon senso, come evidenzia l'espressione scis puto («ritengo che lo sai») che equivale a un «è noto a tutti».

Da sempre i mezzi di trasporto hanno orari di partenza prefissati e quindi ai viaggiatori ritardatari è riservata la sorte di restare a terra. Nel caso del libro del poeta il ritardatario è uno $\operatorname{solo}^{25} \mathrm{e}$, come tale, non ha alcuna possibilità di ottenere un rinvio, sia pure breve, del momento della partenza. Solo e pressato dall'adirato comandante della nave, ormai deve vincere la sua indecisione e salire a bordo. È evidente che qui Marziale si identifica nel comportamento del suo libro: anche lui vorrebbe restare in Italia, ma si sente costretto a partire dalla tacita ingiunzione di un inesorabile e potente comandante.

\section{Referencias bibliográficas}

Braconi, P. (2015), «Alimentazione a miglia zero», en Parisi Presicce, C. y Rossini, O. (eds.), Nutrire l'impero. Storie di alimentazione di Roma e Pompei, Roma, L'Erma di Bretschneider, 85-91.

Buongiovanni, C. (2012), Gli 'Epigrammata Longa' del decimo libro di Marziale, Pisa, ETS. Canobbio, A. (2011), M. Valerii Martialis Epigrammaton liber quintus, Nápoles, Loffredo.

Citroni, M. (1975), M. Valerii Martialis epigrammaton liber I, Florencia, La Nuova Italia.

Citroni, M. (1986), «Le raccomandazioni del poeta: apostrofe al libro e contatto col destinatario», Maia 38, 111-146.

Citroni, M. (1987), s.u. «Marziale», EV, III, Roma, Treccani.

Friedlaender, L. (1886), M. Valerii Martialis Epigrammaton libri, I, Leipzig, Hirzel.

Fusi, A. (2006), M. Valerii Martialis Epigrammaton liber tertius, Hildesheim-Zúrich-Nueva York, Georg Olms Verlag.

Izaac, H. J. (1930), Martial, Epigrammes I, París, Les Belles Lettres.

La Penna, A. (1983), «Una polemica giocosa di Marziale con Virgilio a proposito del Galeso», $A \& R$ 28, 158-161.

Marchesi, C. (1952), Storia della letteratura latina, II, Milán, Principato.

Norcio, G. (1980), Epigrammi di Marco Valerio Marziale, Turín, UTET.

Parroni, P. (1984), «Nostalgia di Roma nell'ultimo Marziale», Vichiana 13, 126-134.

Salanitro, M. (2005), «Marziale e Virgilio (Mart. 6, 3)», $A \& R$ 50, 1-14.

Salanitro, M. (2012), L'arguzia di Marziale, Urbino, Quattroventi.

24 Bilbilis è definita alta, ma il fiume Salone che altrove in Marziale è caratterizzato dalla fredda temperatura (1.49.12 Salone, qui ferrum gelat) o dalle vorticose acque (10.103.2 rapidis quem Salo cingit aquis) qui ha come attributo un possessivo affettivo (tuum) che sottolinea l'appartenenza del libro, e quindi del poeta, alla città spagnola.

25 Non va dimenticato che su unus, parola finale, contrapposta alla parola iniziale nauem, cade l'accento di tutto il verso che contiene la pointe. 
Scàndola, M. (1996), Marco Valerio Marziale, Epigrammi, trad. di M. Scàndola; note di E. Merli, Milán, BUR.

Timpanaro, S. (1978), Contributi di filologia e di storia della lingua latina, Roma, Edizioni dell'Ateneo \& Bizzarri. 